\title{
Numerical investigation of isotropic beam crystallization process under nonuniform cooling
}

\author{
Tatiana Suhodoeva \\ Department of Computational Mathematics and Mechanics \\ Perm National Research Polytechnic University \\ Perm, Russia \\ vmm@pstu.ru
}

\author{
Anna Kamenskikh \\ Department of Computational Mathematics and Mechanics \\ Perm National Research Polytechnic University \\ Perm, Russia \\ anna_kamenskih@mail.ru
}

\author{
Maria Bartolomey \\ Department of Computational Mathematics and Mechanics \\ Perm National Research Polytechnic University \\ Perm, Russia \\ anna_kamenskih@mail.ru
}

\begin{abstract}
The study is directed to conclusion and approbation of the new defining relations that describe arousal and change of stress fields in isotropic material during crystallization, taking into account temperature strains and structural shrinkage. The statement of the crystallization problem and numerical realization of the process by the example of linear and plane statements have been made. The problem of crystallization of a beam of isotropic material under nonuniform cooling with phase transitions has been considered. The authors removed the defining relations describing the occurrence and change of stress fields in an isotropic material during crystallization, taking into account temperature deformations and structural shrinkage. The numerical realization of the problem of crystallization of a beam was made by two methods: a finite difference method and a finite element method. The tendency of accumulation of an error of the finite difference method has been established. The verification of defining relations by the example of the problem of plate nonuniform controlled cooling from temperature above melting point has been made at the second stage of the study. Displacement, stress and strain fields that satisfy the physics of the process and boundary conditions have been obtained.
\end{abstract}

Keywords-crystallization; phase transition; melt; constitutive relations; numerical modeling; boundary element method; finite element method

\section{INTRODUCTION}

Interest in the materials crystallization process appeared more than sixty years ago, the first attempts to describe the behavior of materials during the crystallization process were made at the same time $[1,2,3]$. To remove the constitutive relations and their numerical implementation for mathematical modeling of the problem of materials crystallization is still the important task. Many works of this research are based on removing the constitutive relations and numericalexperimental research of the developed mathematical models. For instance in [4], the problem of describing the thermomechanical behavior of polymer materials in conditions of relaxation conversions is considered in detail. It should be noted that modeling the constitutive relations for describing the crystallization process with the relaxation conversions has wide practical application: in mechanical engineering industry [5], aircraft industry [6], metallurgy [7, 8], microstructure [9] of materials and etc. It is possible to conclude that to obtain and to verify the constitutive relations for the crystallization process with a relaxation conversion are the basis of the solution of the problem of this type and a very laborconsuming process.

There are a lot of works devoted to the study of thermomechanical behavior of polymeric materials, casting billets, ingots and other details, as well as analysis of the crystallization process in the modern technological and engineering. Many models do not take into account the relaxation and phase transformations; it is associated with the complexity of the mathematical formulation and numerical implementation. The basis of the numerical realization is mainly used methods of finite differences, finite and boundary elements. To model problems using software applications also is very popular, but opportunities of these applications are limited by classical mathematical formulations. However, many "new" defining relations of the crystallization taking into account relaxation conversions were implemented for bodies of canonical form.

Based on the analysis of scientific problems, the authors can conclude that obtaining constitutive relations for crystallization of material during the nonuniform cooling of the body and the numerical realization of the obtained relations to model problems are a current problem.

In this paper, the authors consider a model problem of crystallization of a body from isotropic material with nonuniform cooling of the body. The initial state is the melt; it is described as a viscous liquid. The material crystallizes during phase transition; the new structure is a model of a linearly elastic body. The system is a two-component mixture of solid and liquid fraction, the volumetric content of each is determined by the degree of crystallization. 


\section{DEFINING RELATIONS}

The mathematical model describing the occurrence and change of stress fields in an isotropic material during solidification, taking into account temperature deformations and structural shrinkage was considered. The system crystallized from the melt is a two-component mix of the original and the final (fully crystallized) products. The material in the melted state was assumed as viscous liquid, and in crystallized - as a linearly elastic body.

The potential energy of system $F(t)$ is defined as:

$$
F(t)=F_{k r} \alpha(t)+F_{r}(1-\alpha(t))
$$

where $F_{r}$ - melt potential, $F_{k r}$ - crystal fraction potential, $\alpha(\mathrm{t})$ - the degree of the material crystallization; it defines the current volume content of each component at the given time.

Physical relations for crystallized fraction are defined as:

$$
\sigma_{i j}=\lambda \theta \delta_{i j}+2 \mu \varepsilon_{i j}
$$

where $\sigma_{i j}$ and $\varepsilon_{i j}$ - components of stress and strain tensors, $\theta$ - first invariant of strain tensor, $\delta_{i j}-$ Kronecker's symbol.

Physical relations for melt are defined as:

$$
\sigma_{i j}=-p \delta_{i j}+\lambda_{1} \dot{\theta} \delta_{i j}+2 \mu_{1} \dot{\varepsilon}_{i j}
$$

The total stress field of the system, provided that the strains of the melt and crystallized fraction are the same, will receive the substitution of expressions (2) and (3) differentiated expression (1):

$$
\begin{aligned}
& \sigma_{i j}=\alpha(t)\left(\lambda \theta \delta_{i j}+2 \mu \varepsilon_{i j}\right)+ \\
& +(1-\alpha(t))\left(-p \delta_{i j}+\lambda_{1} \dot{\theta} \delta_{i j}+2 \mu_{1} \dot{\varepsilon}_{i j}\right)
\end{aligned}
$$

The stress tensor in expression (4) was obtained without taking into account the dependence of strains on the degree of crystallized fraction increment. Physical relations of the system melt-crystallized body are not applicable for the description of the phase transition; they are a simplified model for further reasoning.

The changing of the elastic potential with the gradual solidification of the melt was considered later. Let at time $t=t_{1}$ subsequent change in the degree of crystallization occur on the magnitude of $\Delta \alpha_{1}$. So the total degree of the increment will be equal to $\Delta \alpha_{0}+\Delta \alpha_{1}$. A volume part of a noncrystallized part that is proportional to $\Delta \alpha_{1}$ turned into solid state and with time $t_{1}$ - will be deformed together with the previously solidified part of the material that is proportional to $\Delta \alpha_{0}$. This approach was implemented in [10]. The free energy of the system at this stage is defined as:

$$
\begin{aligned}
& F\left(\varepsilon_{1}\right)=F_{k r}\left(\varepsilon_{1}\right) \Delta \alpha_{0}+F_{k r}\left(\varepsilon_{1}-\varepsilon_{0}\right) \Delta \alpha_{1}+ \\
& +F_{r}\left(\varepsilon_{1}\right)\left(1-\Delta \alpha_{0}-\Delta \alpha_{1}\right)
\end{aligned}
$$

where $\varepsilon_{1}$ - the linear tensor of the total strain until the next change of the increment degree.

At the n-th step 6 corresponding to time $t_{n}$, the expression takes the form:

$$
\begin{aligned}
& F\left(\varepsilon_{n}\right)=F_{k r}\left(\varepsilon_{1}\right) \Delta \alpha_{0}+F_{k r}\left(\varepsilon_{1}-\varepsilon_{0}\right) \Delta \alpha_{1}+\ldots+ \\
& +F_{k r}\left(\varepsilon_{i}-\varepsilon_{i-1}\right) \Delta \alpha_{i}+\ldots++F_{k r}\left(\varepsilon_{n}-\varepsilon_{n-1}\right) \Delta \alpha_{n}+. \\
& +F_{r}\left(\varepsilon_{n}\right)\left(1-\Delta \alpha_{0}-\Delta \alpha_{1}-\ldots-\Delta \alpha_{i}-\ldots-\Delta \alpha_{n}\right)
\end{aligned}
$$

Because the right part of the last equation is the integral sum, carrying out the limiting transition, it turns out that:

$$
F[\varepsilon(t)]=\int_{0}^{\alpha(t)} F_{k r}[\varepsilon(t)-\varepsilon(\tau)] d \alpha(\tau)+F_{r}[\varepsilon(t)] \cdot(1-\alpha(t))
$$

The obtained equation was differentiated by using the derived dependences of stress-strain for the potential of crystallized fraction and the melt $(2,3)$. And making some mathematical transformations, relation (5) was obtained, which reflects the dependence of stresses of the system on power strains:

$$
\begin{aligned}
& \sigma_{i j}(t)=\alpha(t)\left(\lambda \theta(t) \delta_{i j}+2 \mu \varepsilon_{i j}(t)\right)- \\
& -\int_{0}^{\alpha(t)}\left[\lambda \theta(\tau) \delta_{i j}+2 \mu \varepsilon_{i j}(\tau)\right] d \alpha(\tau)+ \\
& +(1-\alpha(t))\left(-p \delta_{i j}+\lambda_{1} \dot{\theta}(t) \delta_{i j}+2 \mu_{1} \dot{\varepsilon}_{i j}(t)\right)
\end{aligned}
$$

At the next step, it is necessary to take into account thermal strains and structural shrinkage of the material. Power strains, included in the expression (5), will take the form of:

$$
\varepsilon(t)=\varepsilon^{\Pi}(\mathrm{t})-\varepsilon^{T}(t)+\varepsilon^{y c}(t)
$$

where $\varepsilon^{\Pi}$ - full strain, $\varepsilon^{T}-$ thermal strain, $\varepsilon^{y c}-$ strain due to shrinkage. 
Structural shrinkage of the material contributes to the overall deformation only from the time of its inception and remains constant $\varepsilon^{y c}(t)=k$

Thermal strains will take the form:

$$
\varepsilon_{i j}^{T}=\int_{T_{n}}^{T(t)} a_{i j}(\tau) d \tau=\delta_{i j} \int_{T_{n}}^{T(t)} a(\tau) d \tau
$$

where $a$ - coefficient of thermal linear expansion, $T(\mathrm{t})-$ temperature in the current time, $T_{n}$-initial temperature. The second equation is realized because the effect of temperature leads to changing in volume and does not change the shape.

Let us substitute the expression of the power strains to the physical relations (5), and obtain the defining relations for crystallizing material from the melt with the effects of temperature, structural shrinkage is fully reflected in the expression (6):

$$
\begin{aligned}
& \sigma_{i j}(t)=\alpha(t)\left(\lambda\left[\theta^{\Pi}(t)-\theta^{T}(t)+3 \mathrm{k}\right] \delta_{i j}+\right. \\
& \left.+2 \mu\left[\varepsilon_{i j}^{\Pi}(t)-\varepsilon_{i j}^{T}(t) \delta_{i j}+k \delta_{i j}\right]\right)- \\
& -\alpha(t)\left[\lambda\left[\theta^{\Pi}(\tau)-\theta^{T}(\tau)+3 \mathrm{k}\right] \delta_{i j}+\right. \\
& \left.+2 \mu\left[\varepsilon^{\Pi}(\tau)-\varepsilon^{T}(\tau) \delta_{i j}+k \delta_{i j}\right]\right] d \alpha(\tau)+ \\
& +(1-\alpha(t))\left(-p \delta_{i j}+\lambda_{1}\left[\dot{\theta}^{\Pi}(t)-\dot{\theta}^{T}(t)\right] \delta_{i j}+\right. \\
& \left.+2 \mu_{1}\left[\dot{\varepsilon}^{\Pi}(t)-\dot{\varepsilon}^{T}(t) \delta_{i j}\right]\right)
\end{aligned}
$$

So integro-differential equation relations for stresses, strains and strain rates, taking into account the history of deformation for the solid fraction, were obtained.

For the problem definition, it is necessary to select a function that determines the increment of the crystallization degree throughout the phase transition. Expression (7) determines the increment value of crystallized fraction in each moment of the time using the table of Laplace's functions:

$$
\begin{aligned}
& \alpha(t)=\int_{a}^{b} \frac{1}{\sqrt{2 \pi}} e^{-\frac{z^{2}}{2}} d z= \\
& =\int_{0}^{b} \frac{1}{\sqrt{2 \pi}} e^{-\frac{z^{2}}{2}} d z-\int_{0}^{a} \frac{1}{\sqrt{2 \pi}} e^{-\frac{z^{2}}{2}} d z= \\
& =\Phi(b)-\Phi(a)
\end{aligned}
$$

where $\Phi(x)$ - Laplace's function, $z=\left(T(t)-T_{0}\right) / \gamma$, the limits of integration $a=\left(T(t)-T_{0}\right) / \gamma$ and $b=\left(T_{l}-T_{0}\right) / \gamma$.

First the problem of nonuniform controlled cooling of the beam with a temperature above the melting point is considered. Initially the material is in the melted state, then it is subjected to a phase transition and become a fully crystallized body at the final stage. The uniaxial stress state is implemented (fig.1).

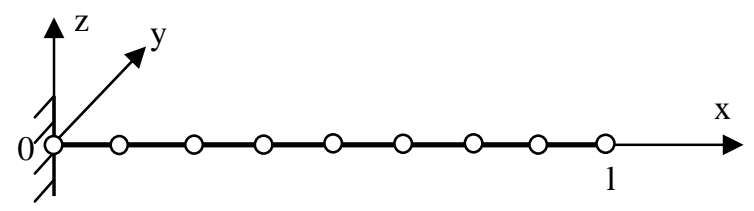

Fig. 1. The model of the prodlem of linear crystallization.

The general mathematical statement includes the equilibrium relations in the absence of body forces and thermal conductivity, physical and geometrical relations, crystallization degree equations, and also initial (8) and boundary (9) conditions:

$$
\begin{gathered}
\left\{\begin{array}{l}
u_{x}(t=0, x)=0 \\
T(t=0, x)=T_{n}
\end{array},\right. \\
\left\{\begin{array}{l}
u_{x}(t, x=0)=0 \\
\sigma_{x x}(t, x=l)=0 \\
\mathrm{~T}_{x}(t, x=0)=0 \\
T(t, x=l)=f(t)
\end{array}\right.
\end{gathered}
$$

At the second stage of the verification of the obtained defining relations, the modeling of the problem of controlled nonuniform cooling of the plate and the temperature above the melting point (Fig.2) was considered. 


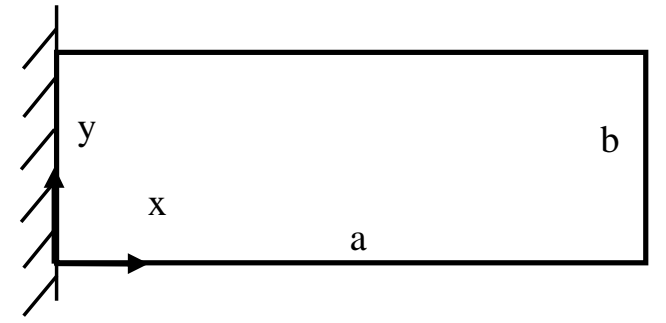

Fig. 2. Scheme of plane problem of crystallization.

The statement of the problem is completed by initial (10) and boundary (11) conditions.

$$
\begin{gathered}
u(t=0, x, y)=0 \\
T(t=0, x, y)=T_{n} \\
\left\{\begin{array}{l}
u_{x}(t, x=0, y)=0 \\
u_{y}(t, x=0, y)=0 \\
\mathrm{~T}_{,}(t, x=0, y)=0 \\
\mathrm{~T}_{,}(t, x, y=0)=0 \\
\mathrm{~T}_{,}(t, x, y=b)=0 \\
T(t, x=a, y)=f(t, y)
\end{array}\right.
\end{gathered}
$$

Spatial-temporal area $0 \leq x \leq a, 0 \leq y \leq b, 0 \leq t \leq T$ was considered, and for its approximation the regular triangular mesh with two unknown displacements in each node was entered. To determine thermal strains and the level of crystallization, the temperature problem with replacing the differential operator of the heat equation finite-difference analog with the implicit scheme of the solution at each semi step was solved.

\section{CONSIDERATION OF THE RESULTS}

The evaluation of the model is conducted within the comparison of numerical solutions of the finite differences (FDM) and the finite element method (FEM) of the problem of beam cooling at a temperature above the melting point. The problem of thermal conductivity is solved initially; the influence of stress-strain state on temperature field during phase transition is not taken into account. Thermophysical and mechanical properties of the material depend on the temperature. Initially the beam is in the melted state; then it is exposed to a phase transition and becomes a fully crystallized body at the final stage. The temperature distribution and degree of crystallization in the beam at each time layer for two variants of numerical implementation of the problem are identical and presented in figures 2.a and 2.b, respectively.
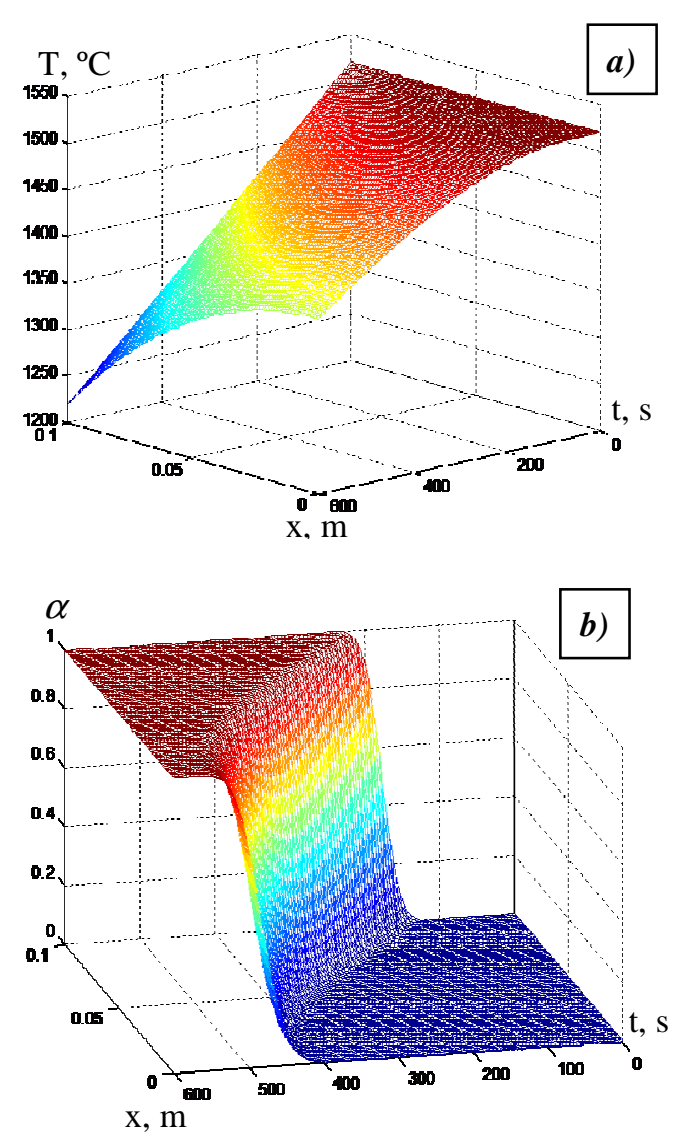

Fig. 3. Temperature field (a) and changes of the degree of crystallization (b) in the beam.

The evaluation of convergence of the solution by the finite difference method (Fig. 3.a) and the finite element method (Fig 3.b) were made in two points of the model (in the middle point and at the free end of the beam) in the framework of the numerical implementation.

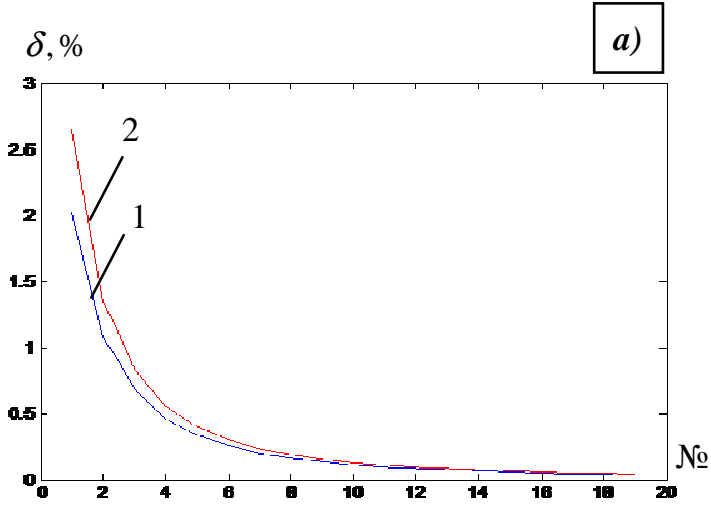




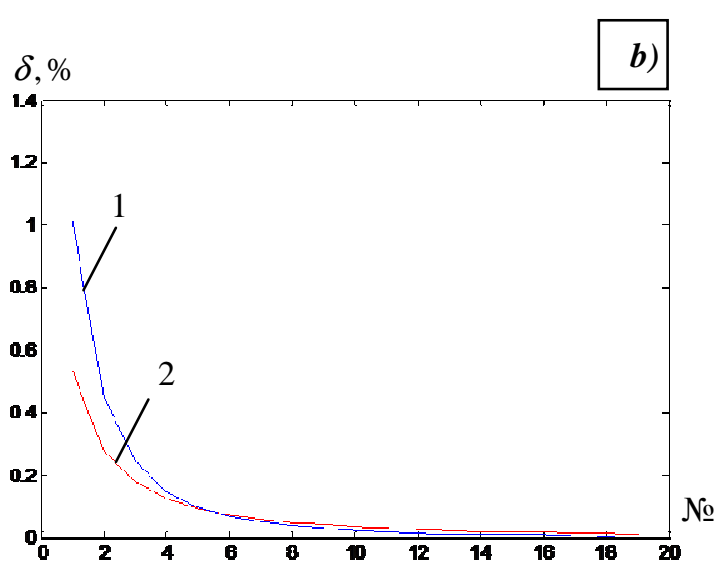

Fig. 4. The convergence of numerical solution with simultaneous regular increase of the partition number at coordinate and time: a) FDM; b) FEM (1 middle of the beam; 2 - free end of the beam)

In figure 3, there is the numerical solution convergence of the problem with simultaneous regular increase the partition number at coordinate and time. But the analysis of the numerical solution of the problem using finite differences method detected the tendency of accumulation of the error. The picture of stress distribution in the numerical solution of the problem by FDM (Fig. 4) is incorrect due to the increase of the negative stresses in the restraint end during the time; only boundary conditions at the free end are carried out. During testing, the cause of the results distortion was revealed: there is accumulation of the error at the full strains that leads to non-zero elastic strains.

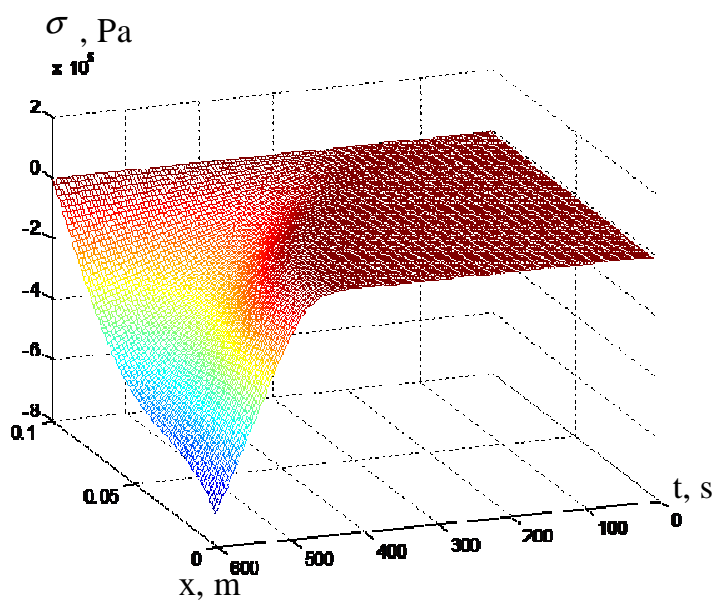

Fig. 5. Stresses along the x-axis.

This error could occur in connection with a simplified approximation scheme: when replacing differential operators by finite-difference analogs, the dependence of the physical variables and the crystallization degree from coordinates was not taken into account. An attempt to fix the error led to the problem of calculating the derivatives of the three functions and the scheme became very bulky. Implementation of the modified method gave the incorrect results and the buckling of the solution was observed.
In connection with the accumulation of the error of numerical solution, using the finite differences method the conclusion to use in further studies only the finite element method for the implementation of the problem of crystallization in the obtained production was made. As the results of numerical modeling by the finite difference method were wrong, the displacements and strains obtained only by the finite element method are shown in figure 5.
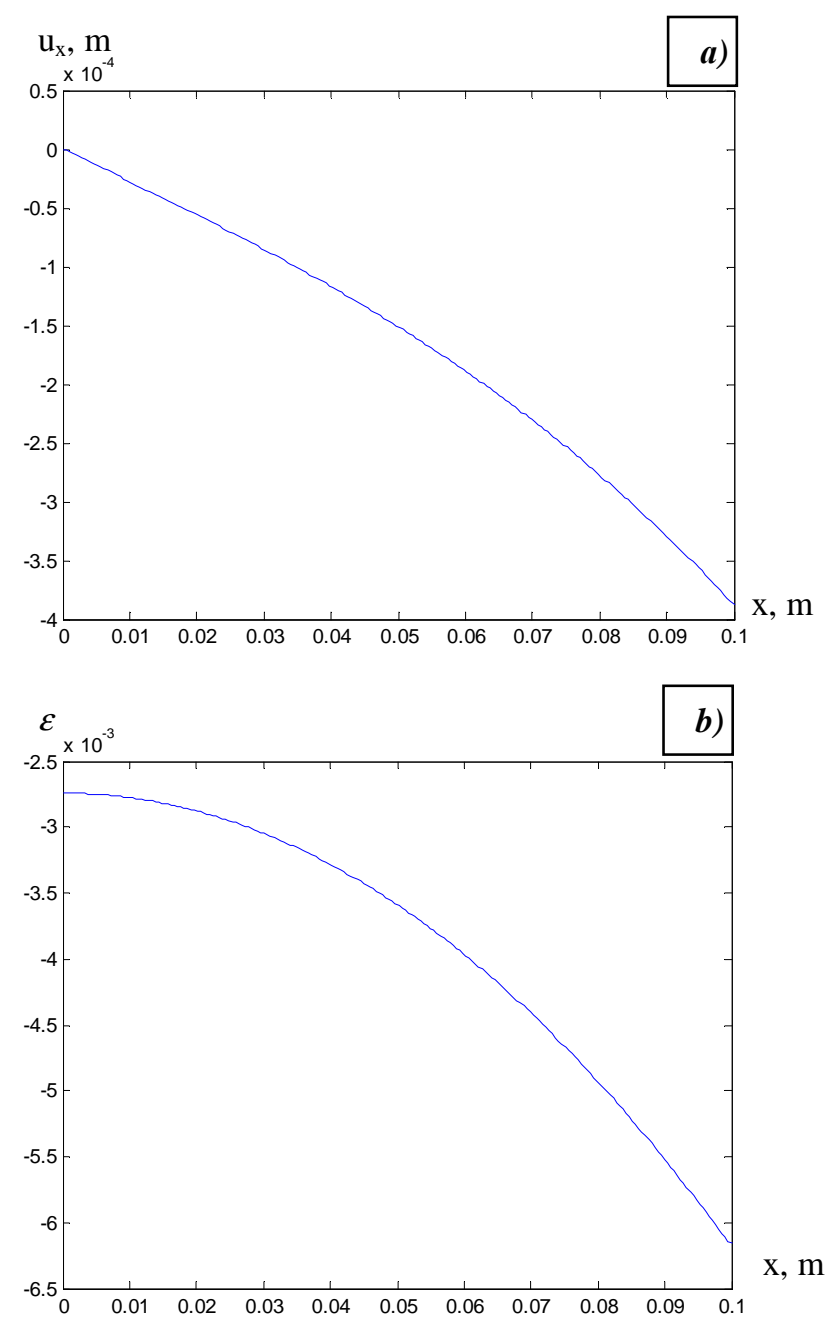

Fig. 6. Displacements (a) and full strains (b) of the beam using FEM.

As the authors supposed, displacements and strain are negative that indicates the beam is in compression, and there is the implementation of all boundary conditions. Displacement, strain and stress fields obtained using the finite element method for a beam of isotropic material at nonuniform cooling do not contradict the physics of the crystallization process.

At the second stage of the problem of crystallization with phase transition based on the obtained equations, the modeling problem of nonuniform cooling of the plate with height $0.1 \mathrm{~m}$ and width $0.2 \mathrm{~m}$ was made. The problem modeling was performed only by the finite element method. The field of temperature in a finite time is picked up so that the temperature of each point of the plate does not exceed the crystallization temperature. Figure 7.a presents the picture of 
approximation of the three-dimensional area. The division into elements was carried out by Delone's triangulation. During cooling of the melt below the temperature of crystallization, the solidification of the plate occurs, the deformed geometry of the structure is shown in figure 7.b.

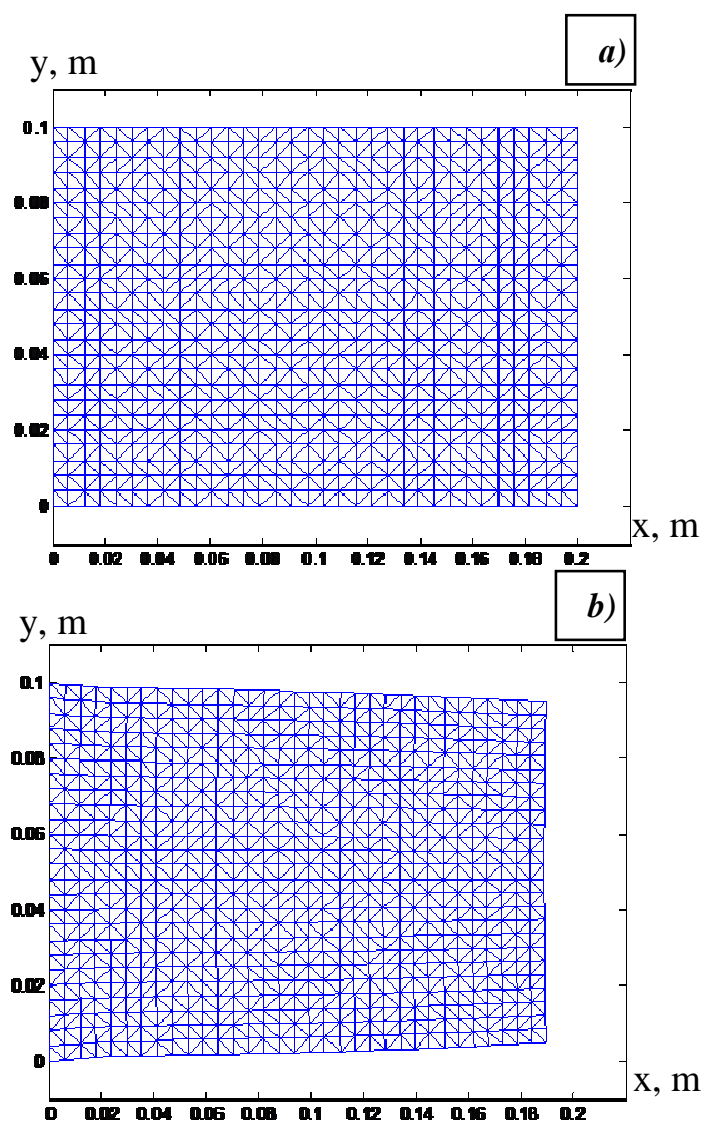

Fig. 7. Nondeformed (a) and deformed (b) plate (displacements of nodes are shown in a tenfold increase)

It should be noted that the plate's deformation picture in the process of crystallization completely satisfies the physics of the process and to the boundary conditions. Stress and strain fields were obtained and convergence of numerical solution was observed in the framework of the problem

Numerical modeling of the crystallization of bodies taking into account temperature strains and structural shrinkage, made in one-dimensional and two-dimensional statement, confirms the capacity of the developed equations.

\section{CONCLUSIONS}

Some results of the solution of the problem of numerical simulation of beam crystallization from isotropic material under nonuniform cooling can be formulated as conclusions:

1. The derivation of new defining relations for describing the emergence and change of stress fields in isotropic material during crystallization, taking into account temperature strains and structural shrinkage, has been performed.
2. The mathematical models that allow describing the process of phase transition in an isotropic material have been constructed based on new physical relations.

3. Full mathematical statement of the problem of crystallization of the beam of isotropic material in linear stress-strain state has been made completely.

4. The numerical implementation of the process of crystallization of the beam of isotropic material under nonuniform cooling by two numerical methods has been realized in Matlab: the finite difference method and the finite element method.

5. The assessment of convergence of the numerical solution of the crystallization problem of the beam of isotropic material under nonuniform cooling has been performed:

- The tendency of accumulation of the error in the numerical solution by the finite difference method, associated with a simplified approximation scheme, has been detected: in case of replacing the differential operators by the finitedifference equivalents, the dependence of the physical variables and the degree of crystallization on coordinates were not taken into account. It has been set that if the problem is corrected, the solution by the finite difference method is diverged.

- The numerical solution of the problem of beam crystallization has been set by the finite element method, which is converged and satisfied for all boundary conditions.

6. Numerical modeling of the problem of nonuniform controlled cooling of the plate at temperature above melting point by finite element method has been implemented: the assessment of numerical solution has been made, deformation behavior of the construction during the shrinkage process has been analyzed, final plate's geometry during crystallization has been obtained, assessment of stress and strain fields has been made, the fulfillment of the boundary conditions during whole crystallization process has been set.

7. The analysis of displacement, stress and strain fields obtained by finite element method has been performed for linear and plane problems.

This work was performed with financial support of the Ministry of Education and Science of the Russian Federation (contract No. 02.G25.31.0168 dated 01.12.2015) within implementation of the RF Government Order No. 218 «On the measures of the State support for development of higher education institutions and organizations implementing integrated projects on high-tech manufacturing build-up».

\section{References}

[1] N.A. Avdonin, Mathematical description of crystallization processes, 1980.

[2] G.F. Balandin, The theory of casting. Fundamentals of thermal theory. The solidification and cooling of castings, 1998.

[3] O.M. Belotserkovsky, Numerical methods in the mechanics of liquids, 1973.

[4] V.P. Matveenko, O.Yu. Smetannikov, N.A. Trufanov, I.N. Shardakov, Thermomechanics polymeric materials in conditions of relaxation transition, 2009. 
[5] N.A. Trufanov, L.R. Shayahmetova, "Tumerically-experimental analysis of the ingot crystallization process", Scientific And Technical Volga Region Bulletin, vol. 5. pp. 291-294, 2015.

[6] N.P. Yaroslavtsev, Physical fundamentals of solidification, 2004.

[7] A.S. Dubrovskaya, "The numerical research of turbine blade solidification and structure formation during cristallization process", Scientific And Technical Volga Region Bulletin, vol. 6, pp. 219-222, 2012.

[8] M. Balcar, R. Zelezny, L. Sochor, P. Fila, L. Martinek, "The development of a chill mould for tool steels using numerical modeling", Mater Technol, vol. 4, pp. 76-82, 2008.
[9] T.P. Chernogorova, P.N. Vabishchevich, "Numerical investigation of solidification processes of cylindrical ingots in a metal mould at variable technological circumstance", Int. J. of Heat and Mass Transfer, vol. 42 pp. 3351-3359, 1999.

[10] T.G. Zavyalova, N.A. Trufanov, "Constitutive relations for a viscoelastic body under crystallization conditions", Journal of Applied Mechanics and Technical Physics, vol. 4, pp. 523-530, 2005. 